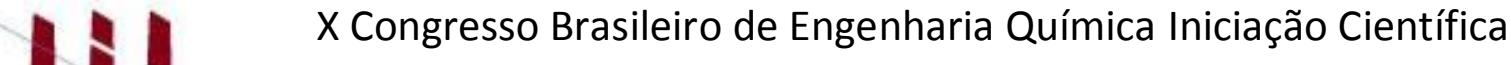 \\ "Influência da pesquisa em Engenharia Química no desenvolvimento tecnológico e industrial brasileiro" \\ Universidade Federal Rural do Rio de Janeiro \\ Universidade Severino Sombra \\ Vassouras - RJ-Brasil
}

\section{ESTUDO NUMÉRICO DA VISCOSIDADE DO FLUXO BIFÁSICO ÓLEO E GÁS NATURAL EM POÇOS DE PETRÓLEO}

\author{
SILVA $^{1}$, L. D. S.; MARINHO ${ }^{2}$, J. L. G.; MEILI ${ }^{2}$, L. \\ ${ }^{1}$ Bolsista de iniciação científica UFAL/CTEC/LCCV ${ }^{2}$ Docente UFAL/CTEC/UAEP \\ Curso de Engenharia Química - Centro de Tecnologia - Universidade Federal de Alagoas \\ Endereço - Av. Lourival Melo Mota, s/n Tabuleiro, Maceió - AL, CEP. 57072-970 \\ email: lucas.ds25@gmail.com
}

\begin{abstract}
RESUMO - O petróleo é um importante combustível fóssil originado da decomposição da matéria orgânica armazenada em sedimentos. Os reservatórios petrolíferos produzem, em sua grande maioria, óleo e gás simultaneamente, os quais fluem até a superfície através de poços. É notório que, dependendo da viscosidade do petróleo, será necessária muita energia para a sua locomoção, gerando um gasto significante. $\mathrm{O}$ presente trabalho tem como objetivo estudar o escoamento de dois tipos de petróleo (um óleo leve e o outro pesado) em dutos verticais. Através de uma simulação numérica, com a utilização um software e modelo matemático adequados, pode-se identificar a influência da queda de pressão assim como o perfil totalmente desenvolvido, possibilitando obter mais informações sobre tipos distintos de petróleo e uma minimização dos custos operacionais do transporte de óleos pesados. As equações governantes escritas no sistema de coordenadas generalizadas foram resolvidas pelo método dos volumes finitos. Todas as simulações foram realizadas usando o aplicativo CFX 13.0. Resultados numéricos das distribuições de queda de pressão e o perfil totalmente desenvolvido do petróleo são apresentados e analisados.
\end{abstract}

Palavras chave: simulação, escoamento vertical, perfil de velocidade.

\section{INTRODUÇÃO}

O escoamento vertical através de poços é uma etapa importante para a produção de petróleo. Em geral, esse escoamento envolve o fluxo bifásico de óleo e gás, uma vez que estes são produzidos simultaneamente em grande parte dos reservatórios de petróleo (Souza, 2009). Óleos obtidos de diferentes reservatórios possuem características diferentes como coloração, densidade e viscosidade. A viscosidade é uma característica que pode influenciar diretamente nos gastos com o transporte de petróleo.

Dependendo da viscosidade do petróleo, será necessária muita energia para a sua locomoção, uma vez que parte dela é perdida durante o escoamento devido ao atrito entre o fluido e a superfície interna do tubo (Fox et al., 2010). Um estudo adequado sobre o comportamento do petróleo em poços permite obter informações sobre tipos distintos de petróleo e pode levar a uma redução 
significativa nos custos operacionais de transporte.

O comportamento do petróleo pode ser representado utilizando métodos analíticos, experimentais ou numéricos. Os métodos analíticos, em geral, são aplicáveis a problemas mais simples, enquanto os métodos experimentais, embora recorram à configuração original do problema, têm sua aplicação dificultada por questões de segurança, tempo e viabilidade econômica (Gonçalves, 2007).

Os métodos numéricos aparecem como uma alternativa viável para resolução de problemas mais complexos, pois apresentam confiabilidade nos resultados, os quais podem ser obtidos de maneira rápida e econômica (Souza et al., 2004). Para problemas envolvendo o escoamento de fluidos, pode-se utilizar uma modelagem matemática baseada nos princípios da conservação de massa, momento linear e energia (Maitelli, 2010).

Com base no que foi apresentado, o presente trabalho tem como objetivo apresentar um estudo numérico em um duto vertical sobre o fluxo bifásico de dois tipos de óleo (um óleo leve e o outro pesado) utilizando o software ANSYS CFX 13.0, onde as equações governantes foram resolvidas pelo método dos volumes finitos.

\section{METODOLOGIA}

\section{Descrição do Modelo}

Para analisar o poço de petróleo, foi considerado que este, pelo seu formato cilíndrico, seria representado de forma simplificada por um tubo vertical de $10 \mathrm{~m}$ de altura e $0,18 \mathrm{~m}$ de diâmetro, conforme ilustrado na Figura 1.

$10 \mathrm{~m}$

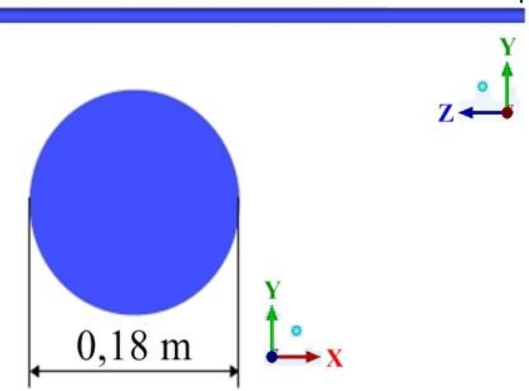

Figura 1 - Visão lateral e frontal do tubo. Modelagem Matemática
Para a resolução do problema, foi utilizada uma modelagem matemática baseada nos princípios de conservação da massa, momento linear e energia, considerando as seguintes hipóteses: escoamento isotérmico e incompressível, regime permanente, efeito da gravidade, domínio tridimensional em coordenadas cartesianas, propriedades físicoquímicas constantes e sem reações químicas.

Como o escoamento é isotérmico, a equação de conservação de energia não foi utilizada.

Para a modelagem, foi adotado o modelo de partícula (abordagem Euleriana-Euleriana). As principais equações que descrevem esse modelo são as seguintes (Marinho, 2012):

$$
\begin{aligned}
\nabla \cdot\left(r_{\alpha} \rho_{\alpha} \vec{U}_{\alpha}\right)=0 & \\
\nabla \cdot\left[r_{\alpha}\left(\rho_{\alpha} \vec{U}_{\alpha} \otimes \vec{U}_{\alpha}\right)\right] & =-r_{\alpha} \nabla P+\nabla \cdot\left[r_{\alpha}\left(\tau_{\alpha}+\tau_{\alpha}^{\text {turb }}\right)\right] \\
& +\vec{S}_{M S \alpha}+\vec{M}_{\alpha}
\end{aligned}
$$

onde as Equações 1 e 2 são as equações de conservação da massa e do momento linear, respectivamente. O termo $\vec{S}_{M S \alpha}$ na Equação 2 descreve as forças de momento devido às forças externas e o termo $\vec{M}_{\alpha}$ descreve as forças interfaciais agindo na fase $\alpha$ devido à presença de outras fases, que pode ser calculado usando a Equação 3:

$\vec{M}_{\alpha}=C_{D} \rho_{\alpha \beta} A_{\alpha \beta}\left|\vec{U}_{\beta}-\vec{U}_{\alpha}\right|\left(\vec{U}_{\beta}-\vec{U}_{\alpha}\right)$

onde o $C_{D}$ é o coeficiente de arraste e $A_{\alpha \beta}$ é a densidade da área interfacial, que pode ser obtida em função da fração da fase dispersa $r_{\beta}$ e do diâmetro principal das partículas $d_{\beta}$, conforme a Equação 4.

$$
A_{\alpha \beta}=\frac{6 r_{\beta}}{d_{\beta}}
$$

Foi utilizado o modelo de turbulência $\kappa$ $\varepsilon$, por permitir uma boa relação entre esforço computacional e precisão (Marinho, 2012). As Equações 5 e 6 descrevem esse modelo: 


$$
\begin{aligned}
\nabla(\rho \vec{U} \kappa)=\nabla & {\left[\left(\mu+\frac{\mu_{t}}{\sigma_{\kappa}}\right) \nabla \kappa\right]+\left(P_{\kappa}-\rho \varepsilon\right) } \\
\nabla(\rho \vec{U} \varepsilon)=\nabla & {\left[\left(\mu+\frac{\mu_{t}}{\sigma_{\varepsilon}}\right) \nabla \varepsilon\right] } \\
& +\frac{\varepsilon}{\kappa}\left(C_{\varepsilon 1} P_{\kappa}-C_{\varepsilon 2} \rho \varepsilon\right)
\end{aligned}
$$

Maiores detalhes sobre a modelagem utilizada podem ser obtidos na tese de Marinho (2012) ou no manual do software ANSYS CFX 13.0.

\section{Geração da Geometria e da Malha}

A geometria e a malha foram geradas no software ANSYS ICEM CFD. A geometria utilizada é composta por uma entrada, uma saída e a parede interna do tubo. A malha construída é constituída por aproximadamente 300 mil elementos hexaédricos e tetraédricos. A Figura 2 mostra a região de saída da malha numérica.

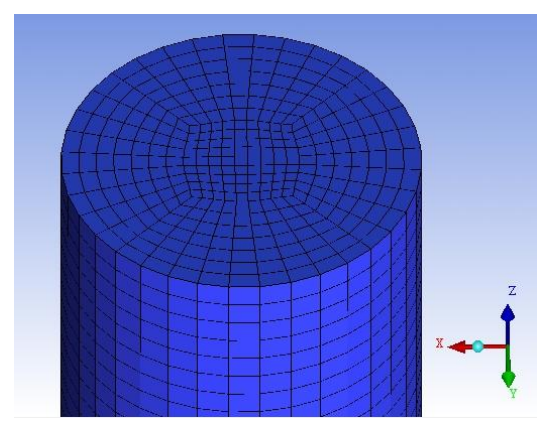

Figura 2 - Representação da saída da malha numérica utilizada nas simulações.

\section{Condições de Contorno}

A Tabela 1 apresenta as condições de contorno utilizadas. O coeficiente de arraste utilizado foi de $C_{D}=0,44$ e foi adotado o valor de $2 \cdot 10^{-3} \mathrm{~m}$ para o diâmetro principal $\left(d_{\beta}\right)$ das partículas da fase dispersa (gás). Admitiu-se que a perda de temperatura é desprezível na altura analisada.

Tabela 1 - Condições de contorno.

\begin{tabular}{l|c}
\hline \multirow{2}{*}{ Entrada } & $\begin{array}{c}U_{o}=U_{g}=1,0 \mathrm{~m} / \mathrm{s} \\
T=298,15 \mathrm{~K}\end{array}$ \\
& $P=1,01 \cdot 10^{5} \mathrm{~Pa}$ \\
Saída & $T=298,15 \mathrm{~K}$ \\
& $r_{o}=0,95$ \\
Fração & $r_{g}=0,05$ \\
volumétrica & \\
\hline
\end{tabular}

\section{Casos Analisados}

No presente trabalho foram analisados os seguintes casos:

- Caso 1: gás natural e óleo leve;

- Caso 2: gás natural e óleo pesado.

Na Tabela 2 encontram-se as principais propriedades físicas dos fluidos utilizados e, em ambos os casos, utilizaram-se as condições de contorno mostradas na Tabela 1 .

Tabela 2 - Propriedades físicas dos fluidos.

\begin{tabular}{l|c|c|c}
\hline & $\begin{array}{c}\text { Óleo } \\
\text { leve }\end{array}$ & $\begin{array}{c}\text { Óleo } \\
\text { pesado }\end{array}$ & $\begin{array}{c}\text { Gás } \\
\text { natural }\end{array}$ \\
\hline$\rho\left(\mathrm{kg} / \mathrm{m}^{3}\right)$ & 855 & 951 & 0,7236 \\
\hline$\mu\left(\mathrm{N} \cdot \mathrm{s} / \mathrm{m}^{2}\right)$ & $2,1 \cdot 10^{-2}$ & $5,0 \cdot 10^{-1}$ & $1,08 \cdot 10^{-5}$ \\
\hline
\end{tabular}

Fonte: Barbosa et al., 2011; Copergás, 2013.

O número de Reynolds $(R e)$ foi utilizado para verificar o regime de escoamento, calculado utilizando a Equação 7:

$$
R e=\frac{\rho U D}{\mu}
$$

\section{RESULTADOS E DISCUSSÃO}

As Tabelas 3 e 4 apresentam os resultados obtidos para a velocidade máxima real e superficial de cada fase, bem como a queda de pressão no fluxo gás e óleo leve e no fluxo gás e óleo pesado, respectivamente.

Tabela 3 - Resultados obtidos para a mistura gás e óleo leve.

\begin{tabular}{c|c|c}
\hline & Gás natural & Óleo leve \\
\hline$U_{S}(\mathrm{~m} / \mathrm{s})$ & 0,10633 & 1,15326 \\
\hline$U_{R}(\mathrm{~m} / \mathrm{s})$ & 1,46055 & 1,22437 \\
\hline$\Delta P(\mathrm{~Pa})$ & \multicolumn{2}{|c}{80076,5} \\
\hline
\end{tabular}

Tabela 4 - Resultados obtidos para a mistura gás e óleo pesado.

\begin{tabular}{c|c|c}
\hline & Gás natural & Óleo pesado \\
\hline$U_{S}(\mathrm{~m} / \mathrm{s})$ & 0,09957 & 0,98776 \\
\hline$U_{R}(\mathrm{~m} / \mathrm{s})$ & 1,30531 & 1,06921 \\
\hline$\Delta P(\mathrm{~Pa})$ & \multicolumn{2}{|c}{88994,5} \\
\hline
\end{tabular}

Observa-se que a velocidade máxima superficial $\left(U_{S}\right)$ para o óleo foi maior que a do gás natural para ambos os fluxos, uma vez que 
a vazão de gás é menor já que ele está em menor quantidade.

A velocidade máxima real $\left(U_{R}\right)$ leva em consideração a área transversal efetiva do tubo disponível para o fluxo de cada fase. Conforme as Tabelas 3 e 4, o gás apresentou maior velocidade máxima real em ambos os fluxos, uma vez que a área transversal disponível para o fluxo de gás é menor, tendo em vista que este está em menor proporção.

Os perfis de velocidade real de cada fase em cada região do tubo são apresentados nas Figuras 3, 4, 5 e 6 . Próximo à região de entrada, a velocidade de cada fase apresentou um perfil uniforme nos dois casos analisados. Por conta dos efeitos viscosos, a velocidade na região próxima à parede diminuiu ao longo do fluxo. Essa diminuição foi compensada com o aumento da velocidade na região central, onde os efeitos viscosos são mínimos e os efeitos inerciais são mais significativos.

No fluxo gás e óleo leve, houve uma pequena diminuição na velocidade de cada fase entre a região a 3 metros da entrada e a região central do duto, conforme as Figuras 3 e 4. Após isso, houve um aumento na velocidade de cada fase entre a região central e a região a de saída do duto. Esse resultado não é consistente e isso se deve, possivelmente, a problemas na modelagem.

A variação de velocidade máxima entre a região a 3 metros da entrada e a região de saída do duto para esse fluxo não ultrapassou $5 \%$. Adotando esse valor como margem de tolerância, pode-se considerar que a velocidade manteve-se constante, isto é, os perfis de velocidade do gás e do óleo leve estão completamente desenvolvidos a partir de 3 metros da entrada.

Para o fluxo gás e óleo pesado (Figuras 5 e 6), a variação de velocidade entre essas regiões foi maior, mas inferior a 5\%, de modo que os perfis de velocidade do gás e do óleo pesado também estão desenvolvidos a partir de 3 metros da entrada. Não houve diminuição na velocidade de cada fase desse fluxo.

Essa maior variação na velocidade pode ser explicada através do regime de escoamento de cada mistura, onde a mistura gás e óleo leve possui $R e=7328,57>2300$, e a mistura gás e óleo pesado possui $R e=342,36<2300$, indicando um escoamento turbulento $\mathrm{e}$ laminar, respectivamente. No escoamento turbulento, a mistura entre as camadas de fluido é mais intensa e isso faz com que a camada limite cresça mais rápido. Assim, o perfil de velocidade no escoamento turbulento desenvolve-se mais rapidamente.

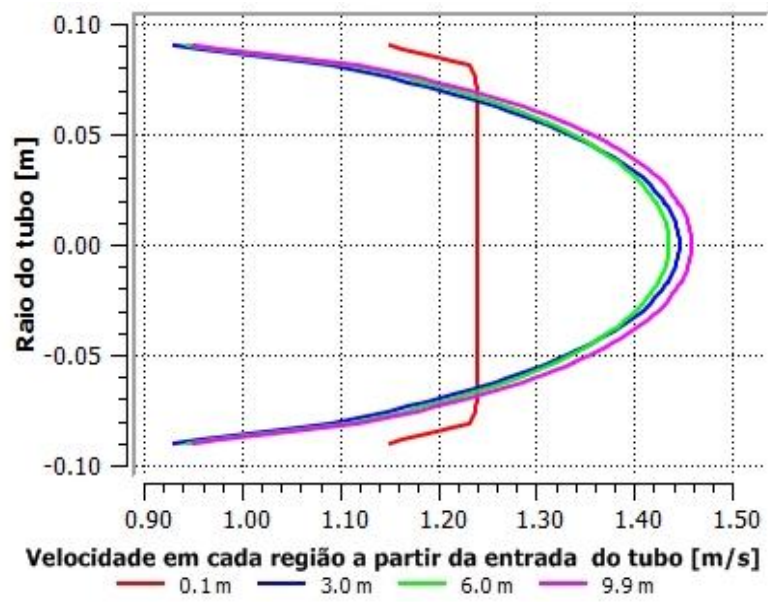

Figura 3 - Perfil de velocidade do gás natural no fluxo gás e óleo leve.

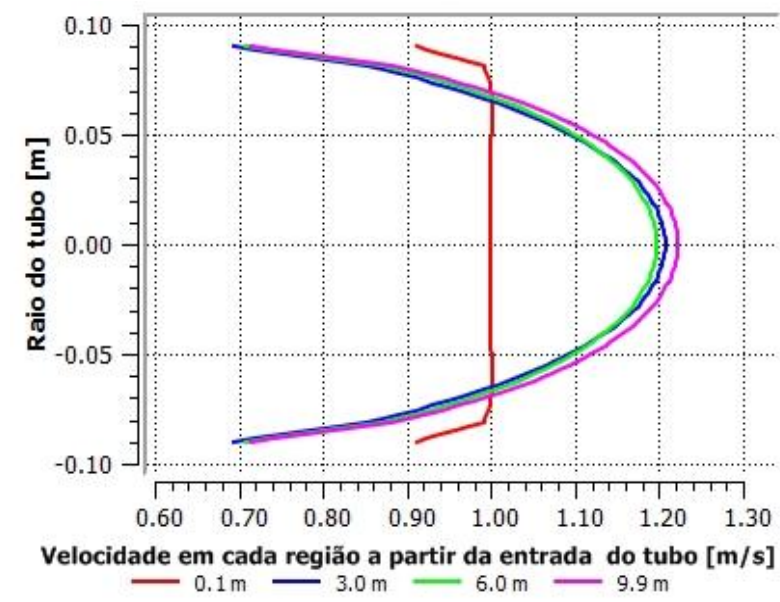

Figura 4 - Perfil de velocidade do óleo leve no fluxo gás e óleo leve.

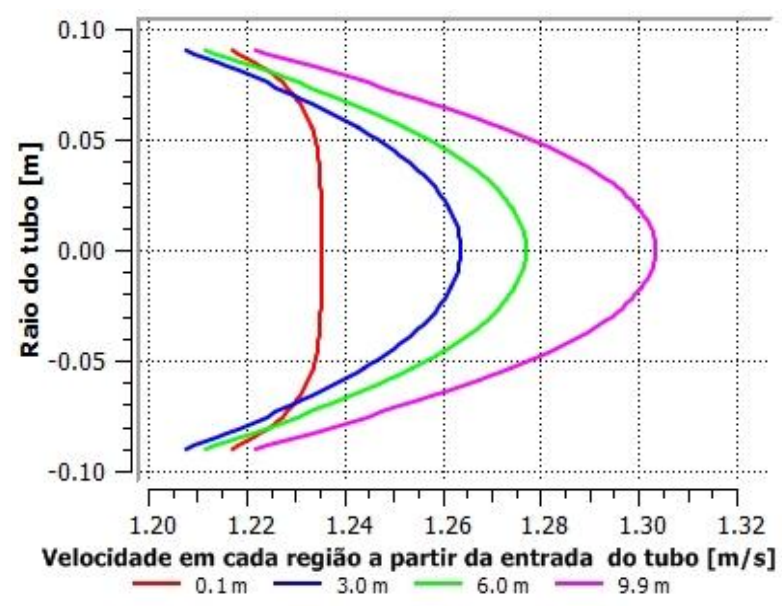

Figura 5 - Perfil de velocidade do gás natural no fluxo gás e óleo pesado. 


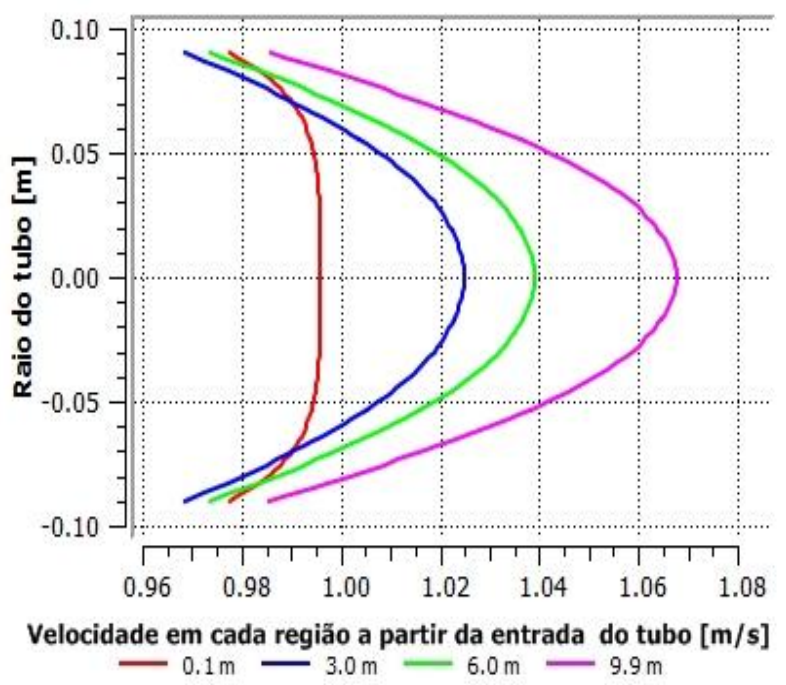

Figura 6 - Perfil de velocidade do óleo pesado no fluxo gás e óleo pesado.

A pressão ao longo tubo para cada mistura é apresentada nas Figuras 7 e 8 e a queda de pressão calculada é exibida nas Tabelas 3 e 4.

Observa-se que a queda de pressão no fluxo gás e óleo pesado foi maior que no fluxo gás e óleo leve. Esse efeito pode ser explicado pelo aumento da viscosidade no fluxo gás e óleo pesado em comparação com o fluxo gás e óleo leve, aumentando a resistência do fluido ao escoar e, consequentemente, as tensões de cisalhamento do fluido, necessitando de mais energia para percorrer a mesma distância.

Isso também pode explicar o fato de as velocidades para a mistura gás e óleo leve terem sido maiores do que as da mistura gás e óleo pesado.

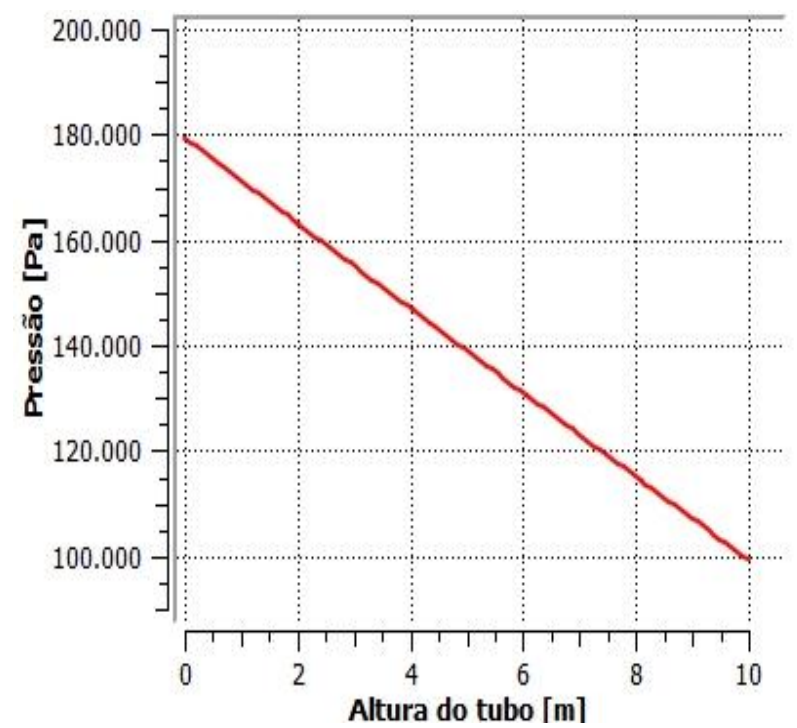

Figura 7 - Pressão no fluxo gás e óleo leve em função da altura do tubo.

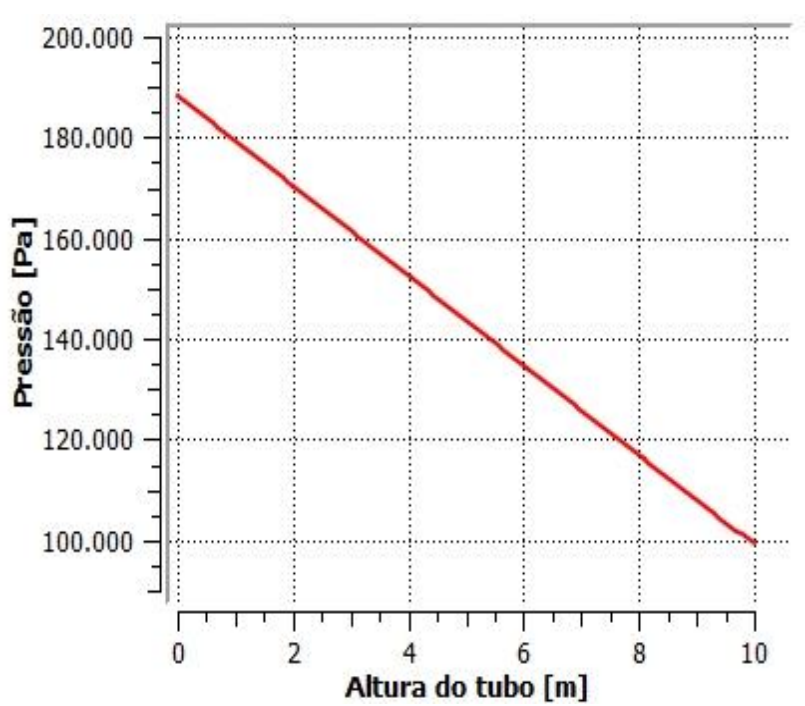

Figura 8 - Pressão no fluxo gás e óleo pesado em função da altura do tubo.

\section{CONCLUSÃO}

A partir da análise dos dados obtidos a após as simulações, foi possível concluir que:

- O fluxo bifásico gás e óleo pesado apresentou uma queda de pressão $11 \%$ maior que a mistura bifásica gás e óleo leve;

- O perfil de velocidade desenvolvido de ambos os casos analisados foi obtido antes da metade do duto. As velocidades obtidas na mistura bifásica gás e óleo leve foram maiores que as velocidades obtidas na mistura bifásica gás e óleo pesado;

- Possivelmente, a modelagem para a mistura gás e óleo leve não está totalmente adequada devido à inconsistência das velocidades na parte central do duto (diminuição da velocidade em comparação à velocidade a $3 \mathrm{~m}$ da entrada seguida de um aumento na velocidade na região de saída do duto).

\section{NOMENCLATURA}

Letras latinas:

\begin{tabular}{|c|c|}
\hline$A_{\alpha \beta}$ & $\begin{array}{l}\text { Densidade da área } \\
\text { interfacial }\end{array}$ \\
\hline$C_{D}$ & Coeficiente de arraste \\
\hline$C$ & $\begin{array}{l}\text { Constante do modelo de } \\
\text { turbulência } \kappa-\varepsilon\end{array}$ \\
\hline & $\begin{array}{l}\text { Constante do modelo de } \\
\text { turbulência } \kappa-\varepsilon\end{array}$ \\
\hline
\end{tabular}

$\mathrm{m}^{-1}$ 


$\begin{array}{llr}C_{p} & \text { Capacidade calorífica a } & \mathrm{J} /(\mathrm{kg} \cdot \mathrm{K}) \\ & \text { pressão constante } & \\ D & \text { Diâmetro do duto } & \mathrm{m} \\ d_{\beta} & \begin{array}{l}\text { Diâmetro principal das } \\ \text { partículas da fase dispersa }\end{array} & \mathrm{m} \\ k & \text { Condutividade térmica } \\ M_{\alpha} & \text { Força de arraste interfacial } & \mathrm{W} /(\mathrm{m} \cdot \mathrm{K}) \\ P & \text { Pressão } & \mathrm{N} / \mathrm{m}^{3} \\ P_{\kappa} & \text { Produção de turbulência } & \mathrm{kg} /\left(\mathrm{m} \cdot \mathrm{s}^{3}\right) \\ R e & \text { Número de Reynolds } & - \\ r_{g} & \text { Fração volumétrica de gás } & - \\ r_{o} & \text { Fração volumétrica de } & - \\ & \text { óleo } & \mathrm{N} / \mathrm{m}^{3} \\ S_{M S \alpha} & \text { Fonte de massa } & \mathrm{K} \\ T & \text { Temperatura } & \mathrm{m} / \mathrm{s} \\ U & \text { Velocidade média } & \mathrm{m} / \mathrm{s} \\ U_{g} & \text { Velocidade média do gás } & \mathrm{m} / \mathrm{s} \\ U_{o} & \text { Velocidade média do óleo } & \mathrm{m} / \mathrm{s} \\ U_{R} & \text { Velocidade máxima real } & \mathrm{m} / \mathrm{s} \\ U_{S} & \text { Velocidade máxima } & \mathrm{Pa} \\ & \text { superficial } & \end{array}$

Letras gregas:

$\begin{array}{llr}\alpha e \beta & \text { Fases envolvidas } & - \\ \varepsilon & \begin{array}{l}\text { Taxa de dissipação } \\ \text { turbulenta }\end{array} & \mathrm{m}^{2} / \mathrm{s}^{3} \\ \kappa & \text { Energia cinética turbulenta } & \mathrm{kg} \cdot \mathrm{m}^{2} / \mathrm{s}^{2} \\ \mu & \text { Viscosidade dinâmica } & \mathrm{N} \cdot \mathrm{s} / \mathrm{m}^{2} \\ \mu_{t} & \text { Viscosidade turbulenta } & \mathrm{N} \cdot \mathrm{s} / \mathrm{m}^{2} \\ \rho & \text { Densidade } & \mathrm{kg} / \mathrm{m}^{3} \\ \rho_{\alpha \beta} & \text { Densidade da mistura } & \mathrm{kg} / \mathrm{m}^{3} \\ \sigma_{\varepsilon} & \text { Constante do modelo de } & - \\ & \text { turbulência } \kappa-\varepsilon & - \\ \sigma_{\kappa} & \text { Constante do modelo de } & \\ & \text { turbulência } \kappa-\varepsilon & \mathrm{kg} /\left(\mathrm{m} \cdot \mathrm{s}^{2}\right) \\ \tau_{\alpha}^{\text {turb }} & \text { Tensões turbulentas de } & \end{array}$

\section{REFERÊNCIAS}

BARBOSA, L. M. C.; LIMA, A. G. B.; NETO, S. R. F. "Escoamento, nãoisotérmico e transiente, de óleo pesado e água em duto vertical com vazamento". Anais do $6^{\circ}$ Congresso Brasileiro de Pesquisa e Desenvolvimento em Petróleo e Gás (PDPETRO), Florianópolis, 2011.

COPERGÁS. Sobre o gás natural. Disponível em: <http://www.copergas.com.br/index.p hp/produtos/>. Acesso em: 17 ago. 2013.

FOX, R. W.; PRITCHARD, P. J.; MCDONALD, A. T. Introdução à mecânica dos fluidos. Rio de Janeiro: LTC, 2010. 7. ed.

GONÇALVES, N. D. F. Método dos volumes finitos em malhas não-estruturadas. Tese (doutorado), Faculdade de Ciências d Universidade do Porto (Portugal), 2007.

MAITELLI, C. W. S. Simulação do escoamento monofásico em um estágio de uma bomba centrífuga utilizando técnicas de fluidodinâmica computacional. Tese (doutorado), Universidade Federal do Rio Grande do Norte, Natal, dez. 2010. p. 4851.

MARINHO, J. L. G. Escoamento anular isotérmico de óleos pesados e água em bifurcações angulares: modelagem e simulação. Tese (doutorado), Universidade Federal de Campina Grande, dez. 2012. p. 29-41.

SOUZA, A. O.; NETO, A. T. P.; ALVES, J. J. N. "Simulação do escoamento gás sólido em risers utilizando o software CFX". Anais do VIII Encontro Latino Americano de Iniciação Científica e IV Encontro Latino Americano de Pós-graduação, São José dos Campos: UniVap, 2004. p. 258261.

SOUZA, R. G. S. Escoamento bifásico líquido-gás: previsão de gradientes de pressão com a correlação de Lockhart \& Martinelli e fluidodinâmica computacional. Projeto final de curso, Universidade Federal do Rio de Janeiro, fev. 2009.

\section{AGRADECIMENTOS}

Os autores agradecem à PETROBRAS pelo apoio financeiro disponibilizado através do LCCV/UFAL, e aos laboratórios LCCV/UFAL e LASSOP/UFAL pelo suporte técnico oferecido. 